Article

\title{
Intravoxel Incoherent Motion Model of Diffusion Weighted Imaging and Diffusion Kurtosis Imaging in Differentiating of Local Colorectal Cancer Recurrence from Scar/Fibrosis Tissue by Multivariate Logistic Regression Analysis
}

\author{
Roberta Fusco ${ }^{1, *(D)}$, Vincenza Granata ${ }^{1}$, Mario Sansone ${ }^{2} \mathbb{D}$, Robert Grimm ${ }^{3} \mathbb{D}$, Paolo Delrio ${ }^{4}$, \\ Daniela Rega ${ }^{4}$, Fabiana Tatangelo ${ }^{5}$ (D), Antonio Avallone ${ }^{6}$, Nicola Raiano ${ }^{1}$, Giuseppe Totaro ${ }^{7}$, \\ Vincenzo Cerciello ${ }^{8}$, Biagio Pecori ${ }^{7}$ and Antonella Petrillo ${ }^{1}$ (D)
}

1 Division of Radiology, “Istituto Nazionale Tumori-IRCCS—Fondazione G. Pascale”, Via Mariano Semmola, 80131 Naples, Italy; v.granata@istitutotumori.na.it (V.G.); n.raiano@istitutotumori.na.it (N.R.); a.petrillo@istitutotumori.na.it (A.P.)

2 Department, Electrical Engineering and Information Technologies DIETI, Via Claudio, 80125 Naples, Italy; msansone@unina.it

3 Siemens Healthcare GmbH, Henkestraße 127, 91052 Erlangen, Germany; robertgrimm@siemens-healthineers.com

4 Division of Colo-rectal Surgical Oncology, "Istituto Nazionale Tumori-IRCCS—Fondazione G. Pascale", Via Mariano Semmola, 80131 Naples, Italy; p.delrio@istitutotumori.na.it (P.D.); d.rega@istitutotumori.na.it (D.R.)

5 Division of Diagnostic Pathology, "Istituto Nazionale Tumori-IRCCS—Fondazione G. Pascale”, Via Mariano Semmola, 80131 Naples, Italy; f.tatangelo@istitutotumori.na.it

6 Division of Gastrointestinal Medical Oncology, "Istituto Nazionale Tumori-IRCCS—Fondazione G. Pascale", Via Mariano Semmola, 80131 Naples, Italy; a.avallone@istitutotumori.na.it

7 Divion of Radiotherapy, "Istituto Nazionale Tumori-IRCCS—Fondazione G. Pascale”, Via Mariano Semmola, 80131 Naples, Italy; g.totaro@istitutotumori.na.it (G.T.); b.pecori@istitutotumori.na.it (B.P.)

8 Health Physics, “Istituto Nazionale Tumori-IRCCS—Fondazione G. Pascale”, Via Mariano Semmola, 80131 Naples, Italy; v.cerciello@istitutotumori.na.it

* Correspondence: r.fusco@istitutotumori.na.it; Tel.: +39-0815903738

Received: 30 October 2020; Accepted: 29 November 2020; Published: 1 December 2020

\begin{abstract}
Purpose: The aim of the study is to evaluate the potential of Intravoxel incoherent motion model of diffusion weighted imaging (DWI) and diffusion kurtosis imaging (DKI) in the differentiation of local colorectal cancer recurrence (LCR) from scar/fibrosis tissue in patients that underwent chemo-radiation therapy followed by the total mesorectal excision (TME) for locally advanced rectal cancer (LARC). Methods: Fifty-six patients were retrospectively included for the image analysis. Diffusion and perfusion parameters were extracted by DWI data (apparent diffusion coefficient (ADC), pseudo-diffusion coefficient (Dp), perfusion fraction (fp), and tissue diffusivity (Dt)) and DKI data (mean of diffusion coefficient (MD) and mean of diffusional Kurtosis). Wilcoxon-Mann-Whitney U test, receiver operating characteristic (ROC) analyses, and area under ROC curve (AUC) were used in a univariate statistical analysis. Backward stepwise multivariate logistic regression analysis was also performed. Results: LCR was found in 34 patients and treatment related changes such as scar/fibrosis tissue in 22 patients. At univariate analysis, low performance was reached by the mean value of Kurtosis with and AUC of 0.72 and an accuracy of $75 \%$, respectively. Considering a regression model obtained as weighted sum of the ADC, Kurtosis, fp and Dp mean values, reached an AUC of 0.82 with a sensitivity of $72 \%$, a specificity of $93 \%$, and an accuracy of $81 \%$.
\end{abstract}


Conclusions: DWI derived parameters combined with DKI derived metrics in a multivariate model could allow differentiating of local colorectal recurrence from scar/fibrosis tissue after TME of LARC.

Keywords: rectal cancer; magnetic resonance imaging; recurrence

\section{Introduction}

The third most common cancer and the third leading cause of oncological death in the United States and Europe is the colorectal cancer [1,2]. Total mesorectal excision (TME) following neoadjuvant chemo-radiation therapy (CRT) is the gold standard for locally advanced rectal cancer (LARC) treatment [3]. One concern after surgery is the local recurrence rate, which although recently improved, is still relevant [4].

Local recurrence in patients with rectal cancer undergoing surgical resection has an incidence of $2.6-30 \%[5,6]$. Total mesorectal excision has standardized the surgical technique in treating primary rectal cancer and has significantly reduced the local colorectal cancer recurrence (LCR) rate from 30\% to $10 \%$ [6]. Sauer et al. reported that the rectal cancer local recurrences incidence within five years was $6 \%$ and $13 \%$, respectively, for patients who received preoperative CRT and postoperative CRT $(p=0.006)[3]$.

Pelvic Magnetic Resonance Imaging (MRI) is a standard diagnostic tool in the follow-up of patients with previous rectal cancer and in case of suspicious of local recurrence. In some patients, the presence of a presacral lesion cannot be sufficiently characterized solely using traditional MRI techniques (T1, T2, etc.). Moreover, small tumors within fibrotic scar tissues are difficult to detect [7].

Several researchers have suggested diffusion-weighted imaging (DWI) to assess treatment response [7-12]. Furthermore, Lambregts et al. [7] as well as Nishie et al. [13] have shown the DWI potential as an additional tool for local rectal cancer recurrence detection.

DWI measures the random Brownian water molecules motion, which is linked to cell density, cell membrane integrity, vascularity, and the viscosity of extracellular fluid [14,15]. These properties can be quantified by means of the individual apparent diffusion coefficient (ADC) using a mono-exponential model or by means of diffusion and perfusion tissue features using a bi-exponential model derived by Intravoxel Incoherent Motion method (IVIM). The IVIM allows the calculation of the pure tissue diffusion coefficient (Dt) that describes water motion in the cellular interstitial space, the pseudo-diffusion coefficient (Dp) that describes blood microscopic flow in the vessels, and the perfusion fraction (fp) that describes the proportion of the diffusion motion [10-17].

The conventional DWI model is based on the assumption that water diffusion follows a Gaussian behavior where water molecules diffuse without any [18]. However, water molecules diffusion within biologic tissues shows non-Gaussian behavior due to the presence of microstructures [18]. Jensen and co-workers proposed a non-Gaussian diffusion model named diffusion kurtosis imaging (DKI) [18]. Using this model, it is possible the extraction of the kurtosis coefficient (K), that measures the deviation of tissue diffusion from a Gaussian model, and the diffusion coefficient (D) with the correction of non-Gaussian bias.

The added value of DWI in the prediction and assessment of tumor response to neoadjuvant CRT in patients with rectal cancer was demonstrated by several authors [7-12,19-23]. Moreover, Grosu et al. [24] assessed the value of ADC in the differentiating of locally recurrent rectal cancer from scar tissue demonstrating that ADC had high accuracy. They, therefore, concluded that Diffusion weighted MRI by means of ADC allows the differentiation of tumor recurrence from scar tissue after surgical resection of rectal cancer.

To our knowledge, until now, there have been no studies published comparing IVIM and DKI derived parameters with the aim to evaluate their potential in the differentiation of local colorectal cancer recurrence from scar/fibrosis tissue in patients that underwent to TME after CRT for locally 
advanced rectal cancer (LARC). Therefore, in this study, we evaluated the performance of IVIM and DKI derived parameters in differentiating of local colorectal cancer recurrence from scar/fibrosis tissue using both the univariate statistical analysis and the multivariate ones with a backward stepwise multivariate logistic regression approach.

\section{Materials and Methods}

\subsection{Dataset Characteristics}

This retrospective study is approved by the National Cancer Institute of Naples institutional review board. The study was made in accordance with local relevant guidelines and regulations. Each patient has signed consent to data processing. We searched among the internal surgical database and the radiological information system (Elefante.NET of Agfa Healthcare, Milano, Italy) of our institution from January 2011 to September 2018 an in order to select patients that after CRT followed by total mesolectal excision have suspicious local colorectal cancer recurrence and underwent MR study with DWI sequence. In total, 69 patients were found. Thirteen among 69 (18.8\%) patients were excluded for absence of DWI sequence in MR protocol and then 56 patients (19 female, 37 male) were included in the analysis. Median age was 62.5 years (range $35-82$ years). All patients (T3-4, N+) received a neoadjuvant CRT and then underwent surgical excision. Details on CRT was reported in [20-23].

Recurrent rectal cancer was found in 34 patients and treatment-related changes such as scar/fibrosis tissue in 22 patients. Table 1 reports the patients characteristics.

Table 1. Characteristics of the study population.

\begin{tabular}{cc}
\hline Patients Description & Numbers (\%)/Range \\
\hline Gender & Men $37(66.1 \%)$ \\
\cline { 2 - 2 } Age & Women $19(33.9 \%)$ \\
\hline Local recurrence Description & $62.5 \mathrm{y}$; range: $35-82 \mathrm{y}$ \\
\hline Presacral site & $14(41.2 \%)$ \\
\hline Anastomotic site & $7(20.6 \%)$ \\
\hline Rectal lumen & $10(29.4 \%)$ \\
\hline Precoccygeal site & $3(8.8 \%)$ \\
\hline
\end{tabular}

\subsection{MR Imaging Protocol}

MRI including DWI was performed in follow-up examinations with a mean value of 2.72 years (range 1.2 years to 7.5 years) after surgical treatment. We think that the effect of the time on imaging in terms of detecting the scar versus recurrence can be expected.

MRI was performed with a 1.5T scanner (MAGNETOM Symphony, Siemens Healthcare, Erlangen, Germany), phased-array body coil and parallel imaging. Patients were scanned in a supine position on a flat table-top insert. DW images were acquired in axial plan with a single-shot spin-echo diffusion-weighted echo-planar pulse sequence at b-values of $0,50,100,150,300,600,1000 \mathrm{~s} / \mathrm{mm}^{2}$ with the following sequence parameters: TR $2700 \mathrm{~ms}$; TE $83 \mathrm{~ms}$; no phase oversampling; Field of View $270 \mathrm{~mm} \times 230 \mathrm{~mm}$; pixel spacing $1.70 \mathrm{~mm} \times 1.70 \mathrm{~mm}$; slice thickness $4.0 \mathrm{~mm}$; gap between slice $0.0 \mathrm{~mm}$; receiver bandwidth, $1250 \mathrm{~Hz} /$ pixel.

In order to reduce respiratory artefacts, each patient received bowel preparation and antispasmodic medication. The entire MR imaging protocol was reported in detail in [22,23].

Rectal cancer recurrences were histo-pathologically proven in the 34 patients while fibrosis/scar tissue were histo-pathologically proven in 6 patients $(27.3 \%)$ while in 16 patients $(72.7 \%)$ were confirmed 
by the clinical follow-up. The clinical follow-up ranged from 20 to 108 months and consisted of the following visits for 5 years: Every 3 months in the first 2 years, and then every 6 months in the following 3 years: Visit with rectal exploration; rectoscopy; CEA and blood chemistry routine; 4 . MRI every 6 months for 5 years; annual thoracic abdominal CT scan; colonoscopy after 1 year from treatment; if negative, after 3 years ( 4 years after treatment); if negative, every 5 years.

\subsection{Data Analysis}

A radiologist with 25 years of experience on MR pelvic imaging performed the manual segmentation. Regions of interest (ROIs) were obtained drawing manually by the same radiologist, using the diffusion-weighted images with the highest b-value (Figure 1), along the tumor contours, slice by slice in order to obtain the entire volume of interest.

\section{DWI Features}

For each voxel,12 features were derived by DWI data using the mono-exponential model, the Intra-Voxel Incoherent Motion model with the conventional bi-exponential fitting based on Levenberg Marquardt algorithm (using multiple low b-values $<200 \mathrm{~s} / \mathrm{mm}^{2}$ and higher b-values: 300, $600,1000 \mathrm{~s} / \mathrm{mm}^{2}$ ) and the Diffusion Kurtosis Imaging model (using one low b-value $50 \mathrm{~s} / \mathrm{mm}^{2}$ and multiple high b-values $>200 \mathrm{~s} / \mathrm{mm}^{2}: 150,300,600,1000 \mathrm{~s} / \mathrm{mm}^{2}$ ).

DWI signal decay is most commonly analyzed using the mono-exponential model [14,15]:

$$
A D C=\frac{\ln \frac{S_{0}}{S_{b}}}{b_{1}-b_{0}}
$$

where $S_{b}$ is the MRI signal intensity with diffusion weighting $b, S_{0}$ is the non-diffusion-weighted signal intensity, $b_{1}$ and $b_{0}$ are the gradient factors of sequences $S_{b}$ and $S_{0}$, respectively, and $A D C$ is the apparent diffusion coefficient.

A bi-exponential model using Levenberg Marquardt fitting algorithm was used to estimate IVIM derived parameters that include pseudo-diffusivity $(D p)$, perfusion fraction $(f p)$, tissue diffusivity $(D t)$, and basal signal $S_{0}$ :

$$
\frac{S_{b}}{S_{0}}=f_{p} \cdot \exp \left(-b \cdot D_{p}\right)+\left(1-f_{p}\right) \cdot \exp \left(-b \cdot D_{t}\right)
$$

Multi-b DW images were obtained by voxel-wise fitting using the diffusion kurtosis signal decay Equation (3) by a two-variable linear least-squares algorithm as used in previous [18]:

$$
\frac{S_{b}}{S_{0}}=\exp \left(-b \cdot D+\frac{1}{6} b^{2} \cdot D^{2} \cdot K\right)
$$

In this equation, $D$ is a corrected diffusion coefficient; and $K$ is the excess Diffusion Kurtosis coefficient.

DWI (ADC), IVIM ( $f p, D t, D p)$, and DKI (mean of diffusional Kurtosis and MD) maps were obtained using the post-processing software Body Diffusion Toolbox (Siemens Healthcare, Erlangen, Germany). 


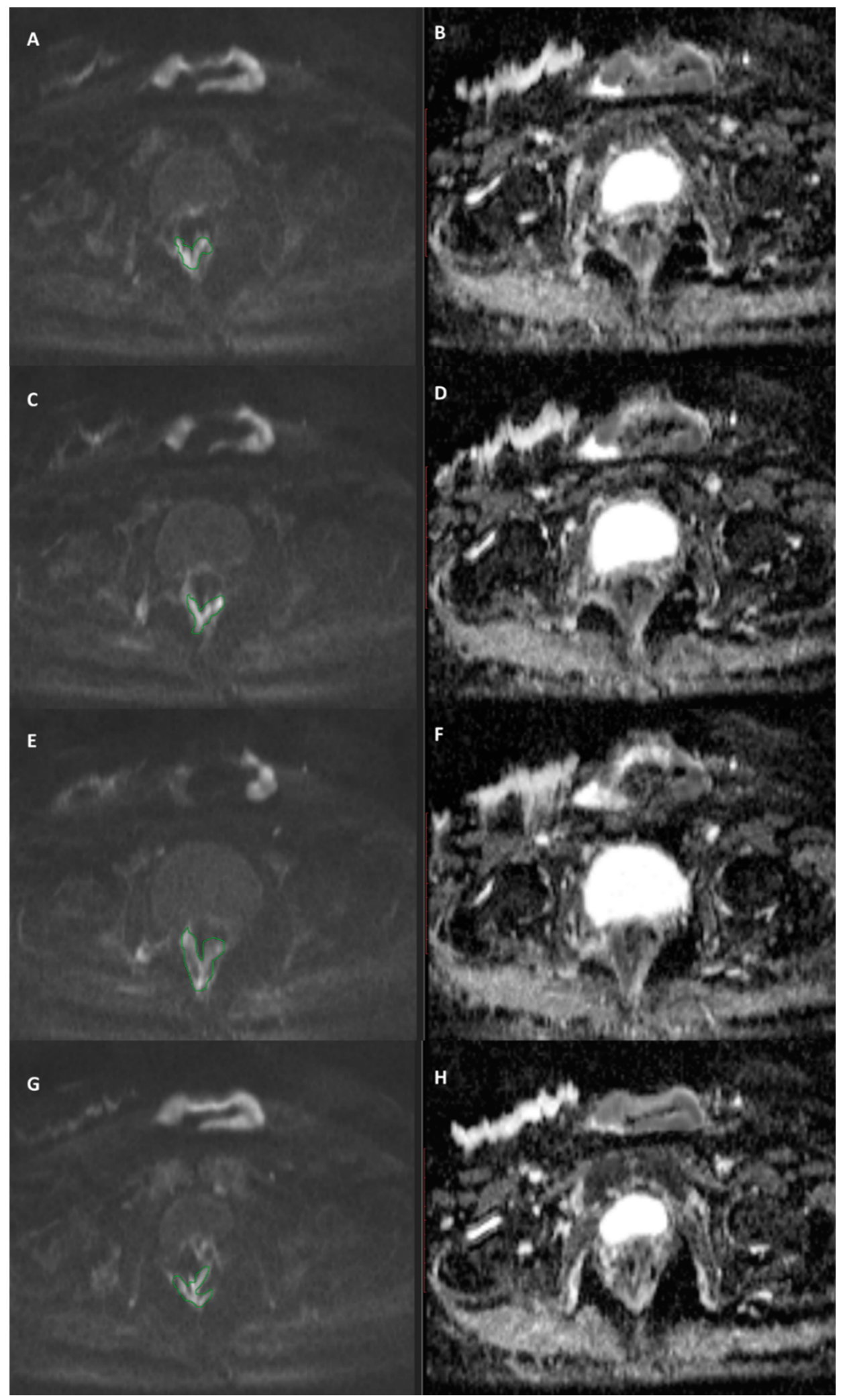

Figure 1. Example of segmentation on diffusion-weighted images with the highest b-value $(\mathbf{A}, \mathbf{C}, \mathbf{E}, \mathbf{G})$ compared with respective ADC maps $(\mathbf{B}, \mathbf{D}, \mathbf{F}, \mathbf{H})$. 


\subsection{Statistical Analysis}

Statistical analysis includes both univariate and multivariate approaches.

\subsubsection{Univariate Analysis}

For each parameter, median and standard deviation values were calculated on the volume of interest and were considered as representative values of presence/absence of LCR. Receiver operating characteristic (ROC) analysis was performed for each parameter and in order to obtain the optimal cut-off value the Youden index was calculated. The Youden index is a function of sensitivity and specificity and is a commonly used measure of overall diagnostic effectiveness in combination with ROC analysis to detect the maximum vertical distance or difference between the ROC curve and the diagonal or chance line; it occurs at the cut-point that optimizes the biomarker-s differentiating ability when equal weight is given to sensitivity and specificity.

Area under ROC curve, sensitivity, specificity, positive predictive value, negative predictive value, and accuracy were obtained. Non-parametric Wilcoxon-Mann-Whitney $U$ test was used in comparisons among groups for continuous variables to identify the parameters that show differences statistically significant between the median values in LCR group versus scar/fibrosis group.

A $p$ value $<0.05$ was considered as significant. Moreover, false discovery rate adjustment (FDR) according to Benjamini and Hochberg for multiple testing was performed.

Statistical analysis was performed using the Statistics Toolbox of Matlab R2007a (MathWorks, Natick, MA, USA).

\subsubsection{Multivariate Analysis}

Backward stepwise multivariate logistic regression analysis was used to assess the association between parameters and the presence of recurrence or fibrosis/scar tissue. Factors for which P values were less than 0.1 in univariate analysis were used as candidate variables for multivariate approach. Stepwise regression is a method of fitting regression models in which the choice of predictive variables is carried out by an automatic procedure; backward elimination, which involves starting with all candidate variables, testing the deletion of each variable using a chosen model fit criterion, deleting the variable (if any) whose loss gives the most statistically insignificant deterioration of the model fit, and repeating this process until no further variables can be deleted without a statistically insignificant loss of fit [25].

\section{Results}

\subsection{Univariate Analysis Results}

A significant discrimination, also considering the false discovery rate (FDR) adjustment, between the group with LCR and the group without LCR was obtained exclusively by the mean value of Kurtosis ( $p$ value $=0.01$ at Wilcoxon-Mann-Whitney $U$ test, see Table 2 ). The Figure 2 reports the ROC curves for DWI and DKI derived parameters to differentiate LCR by scar/fibrosis that show equivalent findings.

Table 3 reports the diagnostic performance for ADC and for the extracted DWI (IVIM) and DKI features to differentiate LCR by scar/fibrosis. Low accuracy was reached by the single parameter to differentiate LCR by scar/fibrosis, however the best results were reached by the mean value of Kurtosis. Mean value of Kurtosis reached an AUC of 0.72 with a sensitivity of $59 \%$, a specificity of $100 \%$, and an accuracy of $75 \%$ (Figure 3). Therefore, univariate analysis results showed that the single diffusion or perfusion parameters was not able with high accuracy to differentiate LCR by scar/fibrosis tissue. 

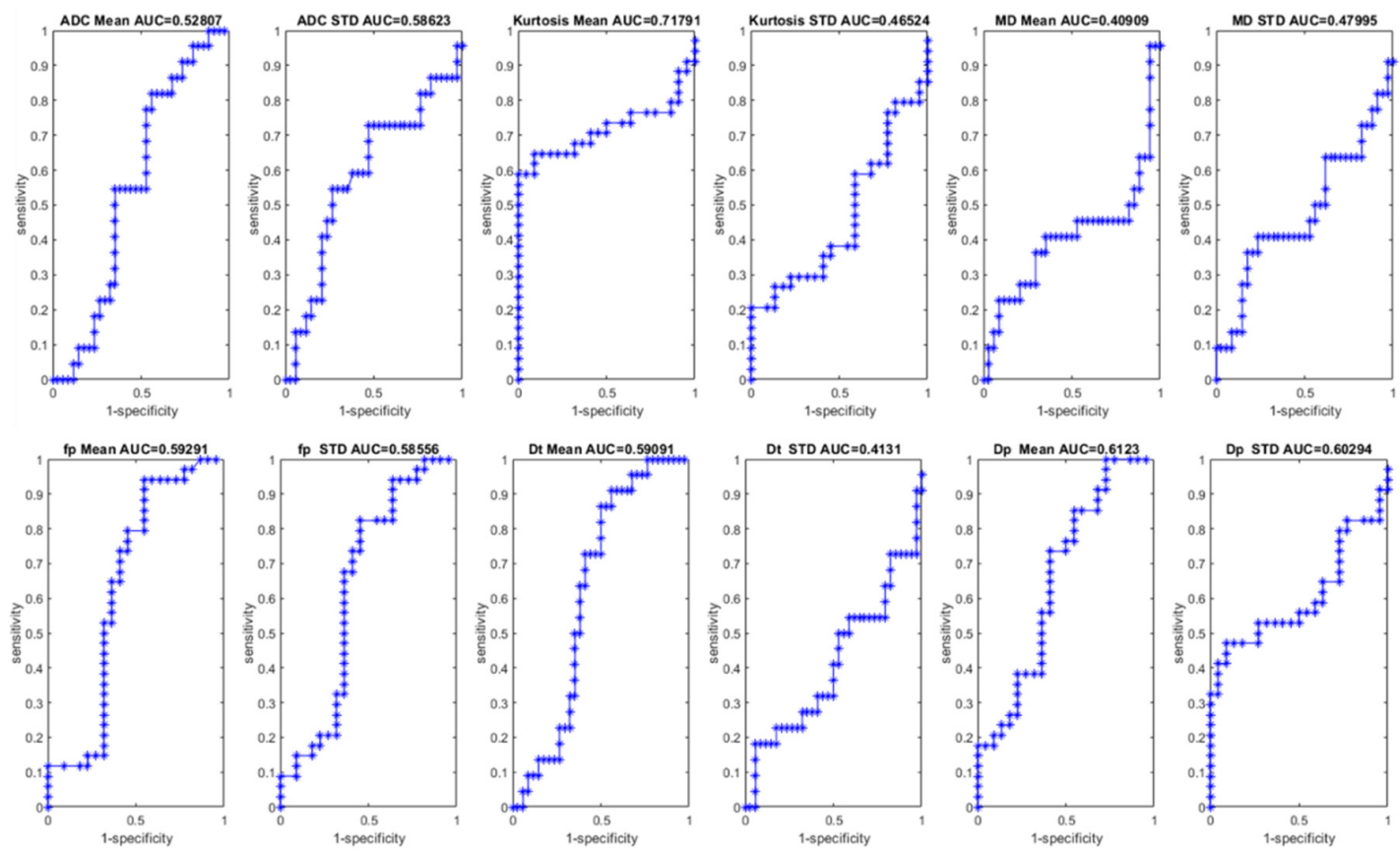

Figure 2. ROC curves for DWI and DKI derived parameters to differentiate LCR by scar/fibrosis. Note. $\mathrm{STD}=$ standard deviation; $\mathrm{ADC}=$ Apparent Diffusion Coefficient; $\mathrm{MD}=$ mean of diffusion coefficient; $\mathrm{fp}=$ perfusion fraction; $\mathrm{Dt}=$ tissue pure diffusion; $\mathrm{Dp}=$ pseudo-diffusion.

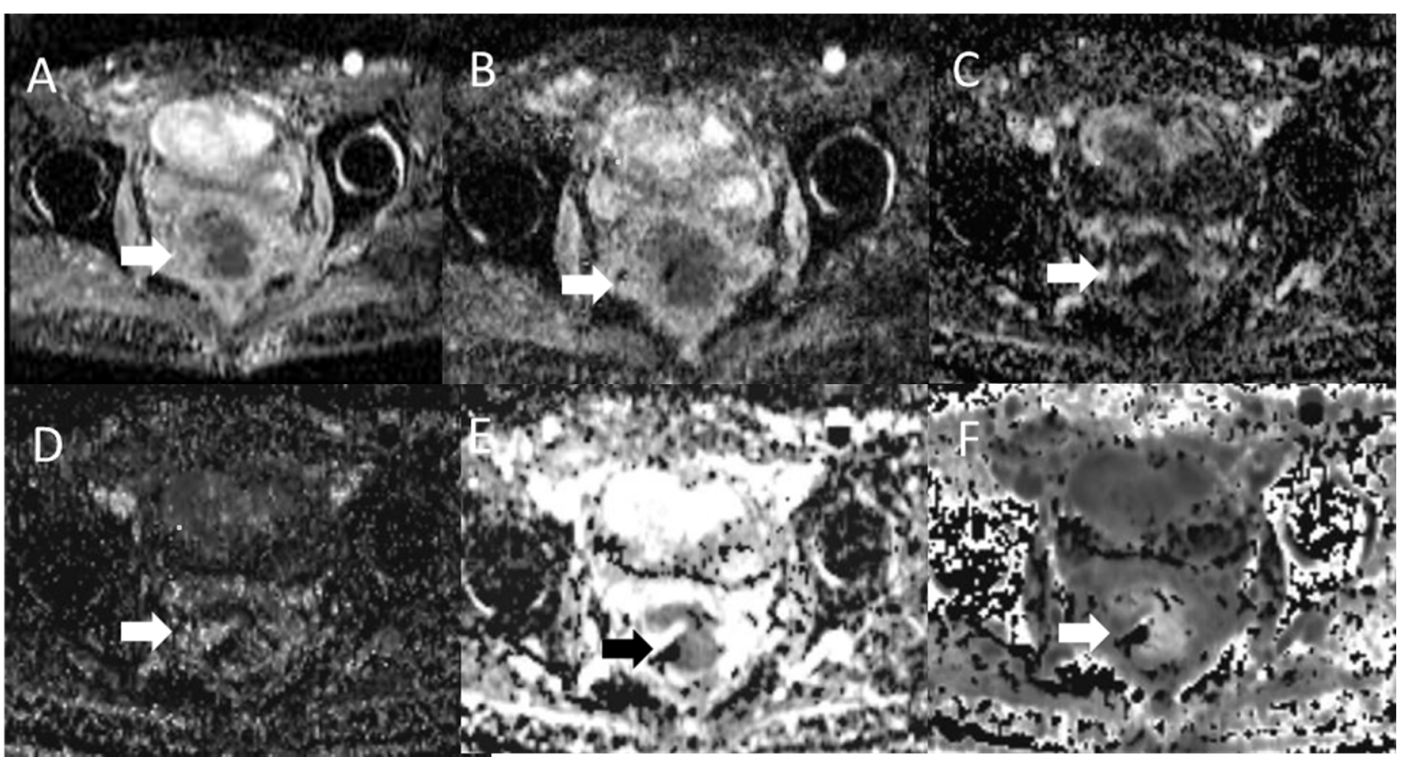

Figure 3. Case with local colorectal cancer recurrence: Apparent diffusion coefficient (ADC) map in (A), Dt map in (B), fp map in (C), Dp map in (D), MD map in (E) and mean of diffusional Kurtosis map in $(\mathbf{F})$. 
Table 2. Median and standard deviation values of diffusion weighted imaging (DWI) and diffusion kurtosis imaging (DKI) derived parameters extracted parameters with and without local colorectal cancer recurrence (LCR).

\begin{tabular}{|c|c|c|c|c|c|c|c|c|c|c|c|c|c|}
\hline & & $\begin{array}{c}\text { ADC Mean } \\
{\left[\mathrm{mm}^{2} / \mathrm{s} \times 10^{-6}\right]}\end{array}$ & $\begin{array}{c}\mathrm{ADC} \text { STD } \\
{\left[\mathrm{mm}^{2} / \mathrm{s} \times 10^{-6}\right]}\end{array}$ & $\begin{array}{c}\text { Kurtosis Mean } \\
{\left[\times 10^{-3}\right]^{*}}\end{array}$ & $\underset{\left[\times 10^{-3}\right]^{*}}{\text { Kurtosis }}$ & $\begin{array}{c}\text { MD Mean } \\
{\left[\mathrm{mm}^{2} / \mathrm{s} \times 10^{-6}\right]}\end{array}$ & $\begin{array}{c}\text { MD STD } \\
{\left[\mathrm{mm}^{2} / \mathrm{s} \times 10^{-6}\right]}\end{array}$ & fp Mean [\%] & fp STD [\%] & $\begin{array}{c}\text { Dt Mean } \\
{\left[\mathrm{mm}^{2} / \mathrm{s} \times 10^{-6}\right]}\end{array}$ & $\begin{array}{c}\text { Dt STD } \\
{\left[\mathrm{mm}^{2} / \mathrm{s} \times 10^{-6}\right]}\end{array}$ & $\begin{array}{c}\text { Dp Mean } \\
{\left[\mathrm{mm}^{2} / \mathrm{s} \times 10^{-5}\right]}\end{array}$ & $\begin{array}{c}\mathrm{Dp} \text { STD } \\
{\left[\mathrm{mm}^{2} / \mathrm{s} \times 10^{-5}\right]}\end{array}$ \\
\hline \multirow{2}{*}{ Scar/Fibrosis } & Median value & 1424.0 & 260.4 & 988.5 & 414.0 & 2305.8 & 499.1 & 13.0 & 70.8 & 1262.6 & 256.4 & 104.0 & 76.4 \\
\hline & STD & 374.7 & 86.3 & 209.9 & 92.6 & 688.7 & 242.6 & 6.9 & 34.9 & 384.4 & 107.8 & 29.3 & 20.1 \\
\hline \multirow{2}{*}{ Recurrence } & Median value & 1122.9 & 202.2 & 1335.1 & 397.5 & 1893.9 & 515.1 & 16.4 & 94.2 & 983.3 & 240.2 & 107.9 & 79.5 \\
\hline & STD & 446.1 & 100.8 & 485.7 & 196.0 & 615.3 & 226.8 & 6.0 & 31.5 & 439.5 & 97.0 & 33.5 & 31.7 \\
\hline \multirow{2}{*}{ Total } & Median value & 1146.6 & 214.0 & 1188.7 & 397.5 & 1941.8 & 515.0 & 16.1 & 93.0 & 1024.3 & 242.7 & 107.8 & 77.3 \\
\hline & STD & 438.8 & 99.0 & 470.2 & 183.3 & 626.6 & 229.1 & 6.2 & 32.1 & 437.1 & 98.7 & 32.8 & 30.2 \\
\hline $\begin{array}{r}p \text { van } \\
\text { Wilcoxon-Man }\end{array}$ & $\begin{array}{l}\text { at } \\
\text { /hitney U test }\end{array}$ & 0.47 & 0.28 & 0.01 & 0.67 & 0.25 & 0.80 & 0.08 & 0.10 & 0.13 & $>0.28$ & 0.05 & 0.20 \\
\hline
\end{tabular}

Note. $\mathrm{STD}=$ standard deviation; $\mathrm{ADC}=$ Apparent Diffusion Coefficient; $\mathrm{MD}=$ mean of diffusion coefficient; $\mathrm{fp}=$ perfusion fraction; $\mathrm{Dt}=$ tissue pure diffusion; $\mathrm{Dp}=\mathrm{pseudo-diffusion}$; * = dimensionless number. In bold were reported the statistically significant parameters in the differentiation of recurrence versus fibrosis/scar tissue. 
Table 3. Diagnostic accuracy of DWI and DKI derived parameters to differentiate LCR by scar/fibrosis.

\begin{tabular}{|c|c|c|c|c|c|c|c|}
\hline & AUC & $\begin{array}{c}\text { Sensitivity } \\
{[\%]}\end{array}$ & $\begin{array}{c}\text { Specificity } \\
{[\%]}\end{array}$ & $\begin{array}{l}\text { PPV } \\
{[\%]}\end{array}$ & $\begin{array}{c}\text { NPV } \\
{[\%]}\end{array}$ & $\begin{array}{c}\text { Accuracy } \\
{[\%]}\end{array}$ & $\begin{array}{l}\text { Cut-off } \\
\text { Value }\end{array}$ \\
\hline $\begin{array}{c}\text { ADC Mean } \\
{\left[\mathrm{mm}^{2} / \mathrm{s} \times 10^{-6}\right]}\end{array}$ & 0.53 & 81.8 & 44.1 & 48.7 & 79.0 & 58.9 & 1054.3 \\
\hline $\begin{array}{c}\text { ADC STD } \\
{\left[\mathrm{mm}^{2} / \mathrm{s} \times 10^{-6}\right]}\end{array}$ & 0.59 & 54.6 & 73.5 & 57.1 & 71.4 & 66.1 & 246.6 \\
\hline $\begin{array}{c}\text { Kurtosis Mean } \\
{\left[\times 10^{-3}\right]^{*}}\end{array}$ & 0.72 & 58.8 & 100.0 & 100.0 & 61.1 & 75.0 & 1184.1 \\
\hline $\begin{array}{c}\text { Kurtosis STD } \\
{\left[\times 10^{-3}\right]^{*}}\end{array}$ & 0.47 & 20.6 & 100.0 & 100.0 & 44.9 & 51.8 & 561.1 \\
\hline $\begin{array}{c}\text { MD Mean } \\
{\left[\mathrm{mm}^{2} / \mathrm{s} \times 10^{-6}\right]}\end{array}$ & 0.41 & 22.7 & 91.2 & 62.5 & 64.6 & 64.3 & 2738.0 \\
\hline $\begin{array}{c}\text { MD STD } \\
{\left[\mathrm{mm}^{2} / \mathrm{s} \times 10^{-6}\right]}\end{array}$ & 0.48 & 36.4 & 82.4 & 57.1 & 66.7 & 64.3 & 699.7 \\
\hline $\begin{array}{c}\text { fp Mean } \\
{[\%]}\end{array}$ & 0.59 & 94.1 & 45.5 & 72.7 & 83.3 & 75.0 & 9.5 \\
\hline $\begin{array}{c}\text { fp STD } \\
{[\%]}\end{array}$ & 0.59 & 82.4 & 54.6 & 73.7 & 66.7 & 71.4 & 7.0 \\
\hline $\begin{array}{c}\text { Dt Mean } \\
{\left[\mathrm{mm}^{2} / \mathrm{s} \times 10^{-6}\right]}\end{array}$ & 0.59 & 86.4 & 50.0 & 52.8 & 85.0 & 64.3 & 981.7 \\
\hline $\begin{array}{c}\text { Dt STD } \\
{\left[\mathrm{mm}^{2} / \mathrm{s} \times 10^{-6}\right]}\end{array}$ & 0.41 & 18.2 & 94.1 & 66.7 & 64.0 & 64.3 & 344.2 \\
\hline $\begin{array}{c}\text { Dp Mean } \\
{\left[\mathrm{mm}^{2} / \mathrm{s} \times 10^{-5}\right]}\end{array}$ & 0.61 & 73.5 & 59.1 & 73.5 & 59.1 & 67.9 & 93.1 \\
\hline $\begin{array}{c}\text { Dp STD } \\
{\left[\mathrm{mm}^{2} / \mathrm{s} \times 10^{-5}\right]}\end{array}$ & 0.60 & 47.1 & 90.9 & 88.9 & 52.6 & 64.3 & 82.4 \\
\hline
\end{tabular}

Note. AUC: Area under ROC curve; PPV: positive predictive value; NPV: negative predictive value; STD = standard deviation; $\mathrm{ADC}=$ Apparent Diffusion Coefficient; $\mathrm{MD}=$ mean of diffusion coefficient; $\mathrm{fp}=$ perfusion fraction; $\mathrm{Dt}=$ tissue pure diffusion; $\mathrm{Dp}=$ pseudo-diffusion; ${ }^{*}=$ dimensionless number. In bold is reported the parameter with highest AUC.

\subsection{Multivariate Analysis Results}

Table 4 reports multivariate regression analysis performed with the parameters that at univariate regression analysis had a $p$ value $<0.1$ (mean value of ADC, Kurtosis, fp and Dp). Using these parameters, we obtained a regression model with a significant finding $(<<0.01)$ to differentiate local recurrence by fibrosis/scar tissue (main contribution was of ADC and Kurtosis mean value).

Considering the multivariate regression model (a weighted sum of the ADC, Kurtosis, fp and Dp mean values that obtained a $p$ value $<<0.01$ ) with the coefficients reported in Table 4 and the optimal cut off value (849.8) calculated by ROC analysis we obtained an AUC of 0.82 with a sensitivity of $72 \%$, a specificity of $93 \%$, and an accuracy of $81 \%$.

Figure 3 shows ADC, DWI, and DKI maps of a patient with a LCR. This figure is purely a visual representation of IVIM and DKI extracted parameters; the differentiation between LCR by scar/fibrosis was made considering the quantitative median value of ROI drawn manually by the expert radiologist and using the optimal cutoff value reported in Table 3.

Figure 4 reports ADC, DWI, and DKI maps of a case with fibrosis tissue after TME. This figure is purely a visual representation of IVIM and DKI extracted parameters; the differentiation between LCR by scar/fibrosis was made considering the quantitative median value of ROI drawn manually by the expert radiologist and using the optimal cut off value reported in the Table 3. 
Table 4. Univariate and multivariate regression analysis results.

\begin{tabular}{|c|c|c|c|c|}
\hline & Univariate Regression Analysis & $\begin{array}{l}\text { Coefficients } \\
\times 10^{-3}\end{array}$ & $\begin{array}{l}\text { Odds } \\
\text { Ratio }\end{array}$ & $p$ Value \\
\hline \multirow{13}{*}{ 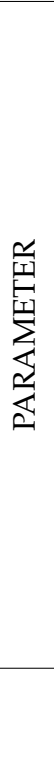 } & ADC Mean & -0.1 & -1.9 & 0.05 \\
\hline & ADC STD & -0.4 & -1.6 & 0.12 \\
\hline & Kurtosis Mean & 0.2 & 3.8 & $<<0.01$ \\
\hline & Kurtosis STD & 0.1 & 0.9 & 0.37 \\
\hline & MD Mean & -0.0 & -0.7 & 0.51 \\
\hline & MD STD & -0.1 & -0.8 & 0.44 \\
\hline & fp Mean & 0.8 & 1.7 & 0.09 \\
\hline & fp STD & 1.3 & 1.5 & 0.13 \\
\hline & Dt Mean & 0.9 & 1.0 & 0.32 \\
\hline & Dt STD & -0.2 & -0.7 & 0.47 \\
\hline & Dp Mean & -0.2 & -2.4 & 0.02 \\
\hline & Dp STD & 1.0 & 1.0 & 0.30 \\
\hline & Multivariate Regression Analysis & $\begin{array}{l}\text { Coefficients } \\
\times 10^{-3}\end{array}$ & $\begin{array}{l}\text { Odds } \\
\text { Ratio }\end{array}$ & $p$ Value \\
\hline \multirow{4}{*}{ 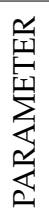 } & ADC Mean & 0.3 & 2.2 & 0.02 \\
\hline & Kurtosis Mean & 0.5 & 3.6 & $<<0.01$ \\
\hline & fp Mean & 0.04 & 0.1 & 0.94 \\
\hline & Dp Mean & -0.4 & -0.5 & 0.63 \\
\hline
\end{tabular}

Note. In bold were reported the statistically significant parameters in the differentiation of recurrence versus fibrosis/scar tissue.

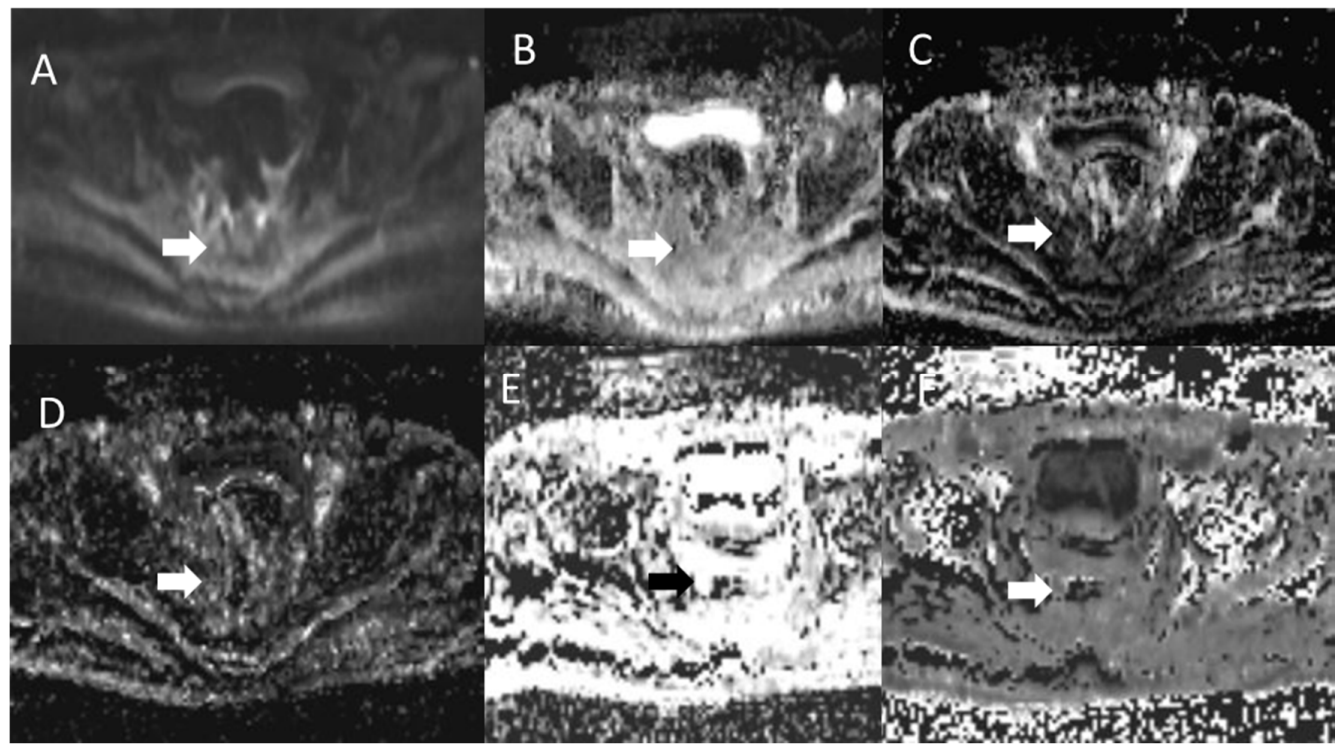

Figure 4. Case with fibrosis tissue after rectal TME: ADC map in (A), Dt map in (B), fp map in (C), Dp map in (D), MD map in (E) and the mean of diffusional Kurtosis map in (F).

\section{Discussions}

The total mesorectal excision introduction and the use of multidisciplinary team in the management of patients affected by locally recurrent rectal cancer has determined a reduction of local colorectal recurrence rate $[11,12]$. 
Several researchers reported in literature that local colorectal recurrence risk is linked to several tumor characteristics such as advanced tumor stage, tumor location in the lower third of the rectum, tumor budding presence, poorly differentiated tumor histology, positive nodal status, of the circumferential resection margin involvement, perineural spread, and extramural venous invasion [25-30]. In addition, the surgical approach plays an essential role in the LCR rate increase. In fact, a mesorectum incomplete resection is linked to a significantly higher risk of five-year local recurrence with respect to those with TME. The local and distant recurrence risk is significantly higher in cases where TME plane is intramesorectal or along muscularis propria, compared to those where TME plane is mesorectal [31-34]. Rectal cancer recurrence is a difficult to treat condition; its earlier identification could help to increase overall survival of colorectal cancer patients [31-34].

MRI is recommended in the patients follow-up who have undergone rectal adenocarcinoma transanal resection, while for patients subjected to TME, Computed Tomography (CT) is the primary modality of follow-up and MR is considered as a problem-solving tool [33-35].

Regarding this, the diffusion-weighted imaging could be considered as quiet a reliable imaging modality for colorectal cancer recurrence, but more studies should be carried out.

Studies have confirmed DWI utility in recurrences detection of other pelvic tumors such as prostate cancer [35], and its value in the response evaluation after neoadjuvant therapy is well documented [11,12,19-23]. DWI has already shown its feasibility in rectal cancer complete responders' detection [20-23]. Molinelli et al. [36] assessed if the addition of gadolinium-enhanced MRI and DWI can improve T2 sequence performance for LCR diagnosis on 43 patients. They reported that, on T2 images, the expert reader achieved an AUC of 0.92 with a sensitivity of $89 \%$, and specificity of $76 \%$ while DWI significantly improved the AUC value to 0.999 . They concluded that DWI increased diagnostic performance for LCR diagnosis compared to T2 imaging alone.

To the best of our knowledge, this is the first study that assess DWI and DKI derived parameters performance in the differentiation of locally recurrent rectal cancer from scar/fibrosis tissue in patients subjected to TME after CRT. Our study showed that a single parameter of diffusion and perfusion was not able to identify local recurrent rectal cancer with high accuracy. However, the best results were obtained using the mean value of Kurtosis with an AUC of 0.72 and an accuracy of $75 \%$. Considering a multivariate regression analysis (with the mean values of $\mathrm{ADC}$, Kurtosis, fp, and Dp), a model mainly weighted by ADC and Kurtosis mean values obtained an AUC of 0.82 with an accuracy of $81 \%$. Therefore, our results support the hypothesis that kurtosis effects could has a better performance in the differentiation of locally recurrent rectal cancer from scar/fibrosis tissue although DKI data were acquired with a maximum $b$ value of $1000 \mathrm{~s} / \mathrm{mm}^{2}$. In general, high $b$-values are suggested in brain applications for the non-Gaussian kurtosis effect assessment [18] while in abdominal applications, for the lower signal-to-noise ratio due to the lower sensitivity of flexible surface coils compared with the head coil and lower T2 relaxation times, very high b-values are not usually applied. Filli et al. [37] reported that the "faster signal decay precludes the application of such high b-values in the body trunk because of 2 effects: (1) the long echo time of the echo-planar imaging sequence due to the longer application of the diffusion gradients and (2) the strong signal attenuation in DWI using high b-values. Furthermore, many tissues exhibit higher ADC values compared with the brain, again resulting in faster signal decay". Some authors have shown that kurtosis effects could be measured in abdominal and whole-body using $800 \mathrm{~s} / \mathrm{mm}^{2}$ as maximum b-values or less at 3T [12,38-41]. We acquired DKI using multiple b-values with a maximum of $1000 \mathrm{~s} / \mathrm{mm}^{2}$ that, coupled with the parallel imaging factor use, resulted in images with acceptable SNR. Our results of univariate analysis were not completely in agreement with the results presented by the Grosu et al. [24] that reported for the ADC value an accuracy of $93 \%$, a sensitivity of $91 \%$, and a specificity of $92 \%$ in the differentiating of locally recurrent rectal cancer from scar tissue with a cut-off ADC value of $1.34 \times 10^{-3} \mathrm{~mm}^{2} / \mathrm{s}$.

Some limitations of the study are the measurement of diffusion and perfusion parameters by manually drawing a ROIs slice by slice along tumor or scar/fibrosis tissue contours; this technique may entail an inter-observer variability. A reproducibility analysis of MR derived parameters was not 
performed, however the use of mean values for extracted parameter by volume of interest allows to obtain more robust measures. Moreover, the number of recruited patients is non-negligible, but not conspicuous. The retrospective nature of study may predispose to selection bias. Considering that the study is retrospective, the radiologists were not blinded by the radiological report, but were blinded by the histological and/or clinical follow-up results. However, it must be clearly stated that the purpose of this study was not to assess the influence of DWI on the diagnostic quality of observers, but to determine DWI ability to discriminate LCR against scar/fibrosis tissue. The calculation of DKI parameters may be suboptimal for the use of an acquisition protocol with $1000 \mathrm{~m} / \mathrm{s}^{2}$ as a maximum $b$ value.

\section{Conclusions}

DWI derived parameters combined with DKI derived metrics using multivariate model could allow differentiating of local colorectal recurrence from scar/fibrosis tissue after total mesorectal excision in patients affected by locally advanced rectal cancer. However, future investigations on large dataset are needed to validate these results.

Author Contributions: Conceptualization, A.P. and R.F.; methodology, R.F.; software, R.G.; validation, A.P., V.G., R.F., and B.P.; formal analysis, R.F.; investigation, A.P., V.G., P.D., F.T., G.T., B.P., A.A., N.R., D.R., and V.C.; data curation, R.F. and M.S.; writing-original draft preparation, R.F. and V.G.; writing-review and editing, A.P. All authors have read and agreed to the published version of the manuscript.

Funding: This research received no external funding.

Conflicts of Interest: The authors as well as the authors' institutions have no actual or potential conflicts of interest including financial, personal or other relationships with other people or organizations that could inappropriately influence (bias) their work. Publication is approved by all authors and by the responsible authorities where the work was carried out.

\section{References}

1. Siegel, R.L.; Miller, K.D.; Jemal, A. Cancer ststistics. CA Cancer J. Clin. 2020, 70, 7-30. [CrossRef] [PubMed]

2. Malvezzi, M.; Bertuccio, P.; Levi, F.; La Vecchia, C.; Negri, E. European cancer mortality predictions for the year 2014. Ann. Oncol. Off. J. Eur. Soc. Med. Oncol. ESMO 2014, 25, 1650-1656. [CrossRef] [PubMed]

3. Sauer, R.; Becker, H.; Hohenberger, W.; Rödel, C.; Wittekind, C.; Fietkau, R.; Martus, P.; Tschmelitsch, J.; Hager, E.; Hess, C.F.; et al. German Rectal Cancer Study Group. Preoperative versus postoperative chemoradiotherapy for rectal cancer. N. Engl. J. Med. 2004, 351, 1731-1740. [CrossRef] [PubMed]

4. Sugarbaker, P.H. Update on the prevention of local recurrence and peritoneal metastases in patients with colorectal cancer. World J. Gastroenterol. 2014, 20, 9286-9291. [CrossRef] [PubMed]

5. Abulafi, A.M.; Williams, N.S. Local recurrence of colorectal cancer: The problem, mechanisms, management and adjuvant therapy. Br. J. Surg. 1994, 81, 7-19. [CrossRef]

6. Cai, Y.; Li, Z.; Gu, X.; Fang, Y.; Xiang, J.; Chen, Z. Prognostic factors associated with locally recurrent rectal cancer following primary surgery (Review). Oncol. Lett. 2014, 7, 10-16. [CrossRef]

7. Lambregts, D.M.J.; Cappendijk, V.C.; Maas, M.; Beets, G.L.; Beets-Tan, R.G.H. Value of MRI and diffusion-weighted MRI for the diagnosis of locally recurrent rectal cancer. Eur. Radiol. 2011, 21, 1250-1258. [CrossRef]

8. Beets-Tan, R.G.H.; Beets, G.L. Rectal Cancer: Review with Emphasis on MR Imaging. Radiology 2004, 232, 335-346. [CrossRef]

9. Curvo-Semedo, L.; Lambregts, D.M.J.; Maas, M.; Thywissen, T.; Mehsen, R.T.; Lammering, G.; Beets, G.L.; Caseiro-Alves, F.; Beets-Tan, R.G.H. Rectal Cancer: Assessment of Complete Response to Preoperative Combined Radiation Therapy with Chemotherapy—Conventional MR Volumetry versus Diffusion-weighted MR Imaging. Radiology 2011, 260, 734-743. [CrossRef]

10. Granata, V.; Fusco, R.; Catalano, O.; Filice, S.; Amato, D.M.; Nasti, G.; Avallone, A.; Izzo, F.; Petrillo, A. Early Assessment of Colorectal Cancer Patients with Liver Metastases Treated with Antiangiogenic Drugs: The Role of Intravoxel Incoherent Motion in Diffusion-Weighted Imaging. PLoS ONE 2015, 10, e0142876. [CrossRef] 
11. Petrillo, A.; Fusco, R.; Granata, V.; Filice, S.; Sansone, M.; Rega, D.; DelRio, P.; Bianco, F.; Romano, G.M.; Tatangelo, F.; et al. Assessing response to neo-adjuvant therapy in locally advanced rectal cancer using Intra-voxel Incoherent Motion modelling by DWI data and Standardized Index of Shape from DCE-MRI. Ther. Adv. Med. Oncol. 2018, 10, 1758835918809875. [CrossRef] [PubMed]

12. Fusco, R.; Sansone, M.; Granata, V.; Grimm, R.; Pace, U.; DelRio, P.; Tatangelo, F.; Botti, G.; Avallone, A.; Pecori, B.; et al. Diffusion and perfusion MR parameters to assess preoperative short-course radiotherapy response in locally advanced rectal cancer: A comparative explorative study among Standardized Index of Shape by DCE-MRI, intravoxel incoherent motion- and diffusion kurtosis imaging-derived parameters. Abdom. Radiol. 2019, 44, 3683-3700.

13. Nishie, A.; Stolpen, A.; Obuchi, M.; Kuehn, D.M.; Dagit, A.; Andresen, K. Evaluation of locally recurrent pelvic malignancy: Performance of T2- and diffusion-weighted MRI with image fusion. J. Magn. Reson. Imaging 2008, 28, 705-713. [CrossRef] [PubMed]

14. Le Bihan, D.; Breton, E.; Lallemand, D.; Aubin, M.L.; Vignaud, J.; Laval-Jeantet, M. Separation of diffusion and perfusion in intravoxel incoherent motion MR imaging. Radiology 1988, 168, 497-505. [CrossRef]

15. Le Bihan, D.; Breton, E.; Lallemand, D.; Grenier, P.; Cabanis, E.; Laval-Jeantet, M. MR imaging of intravoxel incoherent motions: Application to diffusion and perfusion in neurologic disorders. Radiology 1986, 161, 401-407. [CrossRef]

16. Koh, D.-M.; Collins, D.J.; Orton, M.R. Intravoxel Incoherent Motion in Body Diffusion-Weighted MRI: Reality and Challenges. Am. J. Roentgenol. 2011, 196, 1351-1361. [CrossRef]

17. Meng, N.; Bs, X.W.; Sun, J.; Huang, L.; Wang, Z.; Wang, K.; Wang, J.; Ms, D.H.; Wang, M. Comparative Study of Amide Proton Transfer-Weighted Imaging and Intravoxel Incoherent Motion Imaging in Breast Cancer Diagnosis and Evaluation. J. Magn. Reson. Imaging 2020, 52, 1175-1186. [CrossRef]

18. Jensen, J.H.; Helpern, J.A. MRI quantification of non-Gaussian water diffusion by kurtosis analysis. NMR Biomed. 2010, 23, 698-710. [CrossRef]

19. Elmi, A.; Hedgire, S.; Covarrubias, D.; Abtahi, S.; Hahn, P.; Harisinghani, M. Apparent diffusion coefficient as a non-invasive predictor of treatment response and recurrence in locally advanced rectal cancer. Clin. Radiol. 2013, 68, e524-e531. [CrossRef]

20. Cai, G.; Xu, Y.; Zhu, J.; Gu, W.-L.; Zhang, S.; Ma, X.-J.; Cai, S.-J.; Zhang, Z. Diffusion-weighted magnetic resonance imaging for predicting the response of rectal cancer to neoadjuvant concurrent chemoradiation. World J. Gastroenterol. 2013, 19, 5520-5527. [CrossRef]

21. Jung, S.H.; Heo, S.H.; Kim, J.W.; Jeong, Y.Y.; Shin, S.S.; Soung, M.-G.; Kim, H.R.; Kang, H.K. Predicting response to neoadjuvant chemoradiation therapy in locally advanced rectal cancer: Diffusion-weighted 3 tesla MR imaging. J. Magn. Reson. Imaging 2012, 35, 110-116. [CrossRef]

22. Petrillo, A.; Fusco, R.; Petrillo, M.; Granata, V.; Sansone, M.; Avallone, A.; DelRio, P.; Pecori, B.; Tatangelo, F.; Ciliberto, G. Standardized Index of Shape (SIS): A quantitative DCE-MRI parameter to discriminate responders by non-responders after neoadjuvant therapy in LARC. Eur. Radiol. 2015, 25, 1935-1945. [CrossRef] [PubMed]

23. Petrillo, M.; Fusco, R.; Catalano, O.; Sansone, M.; Avallone, A.; DelRio, P.; Pecori, B.; Tatangelo, F.; Petrillo, A. MRI for Assessing Response to Neoadjuvant Therapy in Locally Advanced Rectal Cancer Using DCE-MR and DW-MR Data Sets: A Preliminary Report. BioMed Res. Int. 2015, 2015, 514740. [CrossRef] [PubMed]

24. Grosu, S.; Schäfer, A.-O.; Baumann, T.; Manegold, P.; Langer, M.; Gerstmair, A. Differentiating locally recurrent rectal cancer from scar tissue: Value of diffusion-weighted MRI. Eur. J. Radiol. 2016, 85, 1265-1270. [CrossRef] [PubMed]

25. Efroymson, M.A. Multiple regression analysis. In Mathematical Methods for Digital Computers; Ralston, A., Wilf, H.S., Eds.; Wiley: New York, NY, USA, 1960.

26. Dulk, M.D.; Marijnen, C.A.M.; Putter, H.; Rutten, H.J.T.; Beets, G.L.; Wiggers, T.; Nagtegaal, I.D.; Van De Velde, C.J.H. Risk Factors for Adverse Outcome in Patients with Rectal Cancer Treated with an Abdominoperineal Resection in the Total Mesorectal Excision Trial. Ann. Surg. 2007, 246, 83-90. [CrossRef] [PubMed]

27. Lee, S.-H.; De Anda, E.H.; Finne, C.O.; Madoff, R.D.; Garcia-Aguilar, J. The Effect of Circumferential Tumor Location in Clinical Outcomes of Rectal Cancer Patients Treated with Total Mesorectal Excision. Dis. Colon Rectum 2005, 48, 2249-2257. [CrossRef] [PubMed] 
28. Chan, C.L.; Bokey, E.L.; Chapuis, P.H.; Renwick, A.A.; Dent, O.F. Local recurrence after curative resection for rectal cancer is associated with anterior position of the tumour. Br. J. Surg. 2006, 93, 105-112. [CrossRef]

29. Gunderson, L.L.; Sargent, D.J.; Tepper, J.E.; Wolmark, N.; O’Connell, M.J.; Begovic, M.; Allmer, C.; Colangelo, L.; Smalley, S.R.; Haller, D.G.; et al. Impact of $\mathrm{T}$ and $\mathrm{N}$ stage and treatment on survival and relapse in adjuvant rectal cancer: A pooled analysis. J. Clin. Oncol. 2004, 22, 1785-1796. [CrossRef]

30. Kitz, J.; Fokas, E.; Beissbarth, T.; Ströbel, P.; Wittekind, C.; Hartmann, A.; Rüschoff, J.; Papadopoulos, T.; Rösler, E.; Ortloff-Kittredge, P.; et al. German Rectal Cancer Study Group. Association of Plane of Total Mesorectal Excision with Prognosis of Rectal Cancer: Secondary Analysis of the CAO/ARO/AIO-04 Phase 3 Randomized Clinical Trial. JAMA Surg. 2018, 153, e181607. [CrossRef]

31. Boras, Z.; Kondza, G.; Sisljagić, V.; Busić, Z.; Gmajnić, R.; Istvanić, T. Prognostic factors of local recurrence and survival after curative rectal cancer surgery: A single institution experience. Coll. Antropol. 2012, 36, 1355-1361.

32. Dulk, M.D.; Putter, H.; Collette, L.; Marijnen, C.A.; Folkesson, J.; Bosset, J.-F.; Rödel, C.; Bujko, K.; Påhlman, L.; Van De Velde, C. The abdominoperineal resection itself is associated with an adverse outcome: The European experience based on a pooled analysis of five European randomised clinical trials on rectal cancer. Eur. J. Cancer 2009, 45, 1175-1183. [CrossRef] [PubMed]

33. Hupkens, B.J.P.; Maas, M.; Martens, M.H.; Deserno, W.M.L.L.G.; Leijtens, J.W.A.; Nelemans, P.J.; Bakers, F.C.H.; Lambregts, D.M.J.; Beets, G.L.; Beets-Tan, R.G.H. MRI surveillance for the detection of local recurrence in rectal cancer after transanal endoscopic microsurgery. Eur. Radiol. 2017, 27, 4960-4969. [CrossRef]

34. Colosio, A.; Soyer, P.; Rousset, P.; Barbe, C.; Nguyen, F.; Bouché, O.; Hoeffel, C. Value of diffusion-weighted and gadolinium-enhanced MRI for the diagnosis of pelvic recurrence from colorectal cancer. J. Magn. Reson. Imaging 2014, 40, 306-313. [CrossRef] [PubMed]

35. Roy, C.; Foudi, F.; Charton, J.; Jung, M.; Lang, H.; Saussine, C.; Jacqmin, D. Comparative Sensitivities of Functional MRISequences in Detection of Local Recurrence of Prostate Carcinoma After Radical Prostatectomy or External-Beam Radiotherapy. AJR Am. J. Roentgenol. 2013, 200, W361-W368. [CrossRef]

36. Molinelli, V.; Angeretti, M.G.; Duka, E.; Tarallo, N.; Bracchi, E.; Novario, R.; Fugazzola, C. Role of MRI and added value of diffusion-weighted and gadolinium-enhanced MRI for the diagnosis of local recurrence from rectal cancer. Abdom. Radiol. 2018, 43, 2903-2912. [CrossRef]

37. Filli, L.; Wurnig, M.; Nanz, D.; Luechinger, R.; Kenkel, D.; Boss, A. Whole-body diffusion kurtosis imaging: Initial experience on non-Gaussian diffusion in various organs. Investig. Radiol. 2014, 49, 773-778. [CrossRef]

38. Pentang, G.; Lanzman, R.S.; Heusch, P.; Müller-Lutz, A.; Blondin, D.; Antoch, G.; Wittsack, H.-J. Diffusion kurtosis imaging of the human kidney: A feasibility study. Magn. Reson. Imaging 2014, 32, 413-420. [CrossRef]

39. Wittsack, H.-J.; Lanzman, R.S.; Mathys, C.; Janssen, H.; Mödder, U.; Blondin, D. Statistical evaluation of diffusion-weighted imaging of the human kidney. Magn. Reson. Med. 2010, 64, 616-622. [CrossRef]

40. Granata, V.; Fusco, R.; Sansone, M.; Grassi, R.; Maio, F.; Palaia, R.; Tatangelo, F.; Botti, G.; Grimm, R.; Curley, S.; et al. Magnetic resonance imaging in the assessment of pancreatic cancer with quantitative parameter extraction by means of dynamic contrast-enhanced magnetic resonance imaging, diffusion kurtosis imaging and intravoxel incoherent motion diffusion-weighted imaging. Ther. Adv. Gastroenterol. 2020, 13, 1756284819885052.

41. Granata, V.; Fusco, R.; Setola, S.V.; Palaia, R.; Albino, V.; Piccirillo, M.; Grimm, R.; Petrillo, A.; Izzo, F. Diffusion kurtosis imaging and conventional diffusion weighted imaging to assess electrochemotherapy response in locally advanced pancreatic cancer. Radiol. Oncol. 2019, 53, 15-24. [CrossRef]

Publisher's Note: MDPI stays neutral with regard to jurisdictional claims in published maps and institutional affiliations.

(C) 2020 by the authors. Licensee MDPI, Basel, Switzerland. This article is an open access article distributed under the terms and conditions of the Creative Commons Attribution (CC BY) license (http://creativecommons.org/licenses/by/4.0/). 